\title{
MicroRNA-187 induces diffuse large B-cell lymphoma cell apoptosis via targeting BCL6
}

\author{
FANG HUANG $^{1}$, YAOFENG JIN ${ }^{2}$ and YAFENG WEI ${ }^{1}$ \\ Departments of ${ }^{1}$ Clinical Laboratory and ${ }^{2}$ Clinical Pathology, The Second Affiliated Hospital of Xi'an Jiaotong University, \\ Xi'an, Shaanxi 710004, P.R. China
}

Received March 1, 2015; Accepted January 13, 2016

DOI: $10.3892 / \mathrm{ol} .2016 .4313$

\begin{abstract}
MicroRNAs (miRs) are endogenous non-coding RNAs that serve key functions in a wide range of biological processes, including cell growth, development, apoptosis and carcinogenesis. However, the association between miR-187 and B-cell lymphoma 6 (BCL6) has yet to be fully investigated in lymphoma cell apoptosis. The present study hypothesized that a post-translational mechanism may exist for BCL6 expression, which is regulated by miR-187 in lymphoma cells. The present study demonstrated that the expression of miR-187 in diffuse large B-cell lymphoma (DLBCL) cells was significantly decreased, and its expression was negatively correlated with BCL6 expression. It was also observed that miR-187 directly binds to the 3'-untranslated region of BCL6 mRNA and subsequently suppresses the expression of BCL6. Additionally, the induced expression of miR-187 significantly promoted DLBCL cell apoptosis in vitro. The drug sensitivity of human DLBCL SUDHL2 cells was increased following induction of miR-187 overexpression via an miR-187 mimic. In conclusion, the results of the present study suggest that the modulation of miR-187 expression in DLBCL cells may improve the sensitivity of chemotherapy through BCL6 targeting.
\end{abstract}

\section{Introduction}

Diffuse large B-cell lymphoma (DLBCL) is the most prevalent subtype of non-Hodgkin's lymphoma that develops in adults, accounting for $\sim 40 \%$ of diagnoses resulting from the transformation of follicular lymphoma (1). Despite large improvements in chemotherapy regimens, a substantial percentage of patients $(\sim 40 \%)$ succumb to DLBCL, primarily due to drug resistance and disease relapse (2), thus indicating the urgent requirement for increased specificity chemotherapy.

Correspondence to: Dr Fang Huang, Department of Clinical Laboratory, The Second Affiliated Hospital of Xi'an Jiaotong University, 157 West 5th Road, Xincheng Xi'an, Shaanxi 710004, P.R. China

E-mail: huangfang_01260@163.com

Key words: miRNA-187, B-cell lymphoma 6, diffuse large B-cell lymphoma, apoptosis, multidrug resistance
MicroRNAs (miRNAs/miRs) are non-coding RNAs that function to regulate gene expression in a number of cellular processes, including development, differentiation and proliferation $(3,4)$. During the last decade, miRNAs have gained increasing attention from researchers worldwide due to their crucial functions in human disease and their potential to serve as therapeutic targets. Notably, miR-187 in particular has been reported to be associated with certain types of cancer and other diseases: Zhao et al (5) reported that an increased level of miR-187 inhibits tumor invasiveness during the later stages of carcinogenesis, and Casanova-Salas et al (6) identified that miR-182 and miR-187 may serve as biomarkers for the prognosis of patients with prostate cancer by identifying risk of progression. Locke et al (7) observed that the elevated expression of miR-187 in pancreatic islets from patients with type 2 diabetes was associated with decreased glucose-stimulated secretion of insulin. However, the expression pattern and functions of miR-187 in DLBCL cells has not been identified. Further investigation into miR-187 as a novel therapeutic target may aid the development of a successful therapeutic strategy for patients with DLBCL.

Studies have described B-cell lymphoma 6 (BCL6) as a key regulator of B lymphocyte growth and development $(8,9)$, with modified BCL6 expression implicated in the pathogenesis of DLBCL (10-12). The majority of DLBCL cells maintain a high expression level of BCL6, but the underlying mechanisms that regulate this are not sufficiently understood. In the present study, the association between miR-187 and BCL6 was investigated, alongside the functions of miR-187 in DLBCL cell apoptosis and multidrug resistance.

\section{Materials and methods}

Cell culture, plasmid construction and transfection. The human DLBCL cell lines SUDHL2 and OCI-LY3 and the Burkitt's lymphoma cell line Raji (purchased from Type Culture Collection of the Chinese Academy of Sciences, Shanghai, China) were cultured in RPMI 1640 medium containing $10 \%$ fetal bovine serum. The cells were incubated at $37^{\circ} \mathrm{C}$ in a humidified atmosphere of $5 \% \mathrm{CO}_{2}$ in air. Healthy $\mathrm{B}$ cells were obtained from The Cell Bank of Type Culture Collection of Chinese Academy of Sciences (Shanghai, China). miR-Report BCL6 3'-untranslated regions (UTRs) is the predicted miR-187 binding sites, which 
were commercially constructed by Guangzhou RiboBio Co., Ltd. (Guangzhou, China), and mutation of the potential miR-187 binding sites on the miR-Report BCL6 3'-UTR was performed by Beijing Transgen Biotech Co., Ltd. (Beijing, China). The pcDNA3-BCL6 overexpression plasmid was constructed by GeneChem Co., Ltd. (Shanghai, China), and pcDNA3 was used as the empty vector for control. The scramble and miR-187 mimics were purchased from RiboBio Co., Ltd. The miR-187 mimics are synthesized fragments that share the same sequence as miR-187. The scramble miR was used as a negative control. Transfection was performed using Gene Pulser Xcell ${ }^{\mathrm{TM}}$ Electroporation system (Bio-Rad Laboratories, Inc., Hercules, CA, USA) according to the manufacturer's protocols. The medium was changed with fresh culture medium at 6-8 h post transfection.

Reverse transcription (RT)-quantitative polymerase chain reaction $(q P C R)$. RNA was extracted from the healthy B cells and Raji, OCI-Ly3 and SUGHL2 cells lines using TRIzol ${ }^{\circledR}$ Reagent (Thermo Fisher Scientific, Inc., Waltham, MA, USA) based on the manufacturer's protocols. cDNA was synthesized from $2 \mu \mathrm{g}$ total RNA using the M-MLV Reverse Transcriptase (Promega Corporation) in a $20-\mu 1$ reaction mixture. RT-qPCR was performed using the Applied Biosystems 7300 Real-Time PCR system (Applied Biosystems; Thermo Fisher Scientific, Inc.), with the SYBR ${ }^{\circledR}$ Green Realtime PCR Master mix (Toyobo Co., Ltd., Osaka, Japan) and the appropriate primers. The cDNA was denatured at $95^{\circ} \mathrm{C}$ for $3 \mathrm{~min}$, and subsequently amplification and fluorescence determination were performed in three steps: Denaturation at $95^{\circ} \mathrm{C}$ for $15 \mathrm{sec}$; annealing at $56^{\circ} \mathrm{C}$ for $20 \mathrm{sec}$; and extension at $72^{\circ} \mathrm{C}$ for $20 \mathrm{sec}$. The temperature was decreased to $50^{\circ} \mathrm{C}$ and raised slowly to $95^{\circ} \mathrm{C}$ using a temperature transition rate of $0.1^{\circ} \mathrm{C} / \mathrm{sec}$. The detection of SYBR Green fluorescence, which reflects the amount of double-stranded DNA, was performed at the process of annealing. The amplification cycle number was 45 for all target genes. To discriminate specific from nonspecific PCR products, a melting curve was obtained at the end of each run. All the data were the average of at least three independent experiments. The RT and PCR primers for miR-187 and the endogenous control, U6, were purchased from Guangzhou RiboBio Co., Ltd as follows: miR-187, forward 5'-ACACTC CAGCTGGGGGCCGACGTTGTGTT-3' and reverse 5'-TGG TGTCGTGGAGTCG-3'; U6, forward 5'-CTCGCTTCGGCA GCACA-3' and reverse 5'-AACGCTTCACGAA-3'. Other primers were also purchased from Guangzhou RiboBio Co., Ltd and included the following: BCL6 (76 bp), forward 5'-GGA GTCGAGACATCTTGACTGA-3', reverse 5'-ATGAGGACC GTTTTATGGGCT-3'; 18S (101 bp), forward 5'-ACAACT TTGGTATCGTGGAAGG-3', reverse 5'-GCCATCACGCCA CAGTTTC-3'. Relative mRNA expression levels were calculated as $2{ }^{-\triangle \Delta C q}(13)$ and were normalised against U6.

Western blotting. Cells were homogenized using $1 \mathrm{X}$ Cell Lysis buffer (Cell Signaling Technology, Inc., Danvers, MA, USA) followed by $5-10$ min boiling and centrifugation at 12,000 x g (Eppendorf ${ }^{\circledR}$ Microcentrifuge 5415D; Eppendorf, Hamburg, Germany) to obtain the supernatant. Samples containing $50 \mu \mathrm{g}$ of total protein were separated on a $10 \%$ sodium dodecyl sulfate-polyacrylamide gel electrophoresis gel and transferred onto nitrocellulose membranes (Bio-Rad Laboratories, Inc.). Following saturation with 5\% (w/v) non-fat dry milk in Tris-buffered saline and $0.1 \%(\mathrm{w} / \mathrm{v})$ Tween 20 (TBST; Beijing Biosea Biotechnology Co., Ltd., Beijing, China), the membranes were incubated with the following antibodies at $4{ }^{\circ} \mathrm{C}$ overnight: Rabbit polyclonal anti-caspase-3 (catalog no., sc-7148; dilution, 1:1,000), rabbit polyclonal anti-cleaved-caspase-3 (catalog no., sc-22171-R; dilution, 1:1,000), mouse monoclonal poly ADP ribose polymerase (PARP; catalog no., sc-53643; dilution, 1:500), rabbit polyclonal anti-cleaved-PARP (cPARP; catalog no., sc-23461-R; dilution, 1:500), mouse monoclonal anti-BCL-6 (catalog no., sc-56625; dilution, 1:1,000) and goat polyclonal anti-glyceraldehyde 3-phosphate dehydrogenase (GAPDH; catalog no., sc-48166; dilution, 1:2,000) (Santa Cruz Biotechnology, Inc., Dallas, TX, USA); rabbit polyclonal anti- $\beta$-actin antibody (catalog no., AP0733; dilution, 1:5,000; Bioworld Technology, Inc., St. Louis Park, MN, USA). Following 3 washes with TBST, the membranes were incubated with donkey anti-mouse immunoglobulin ( $\mathrm{Ig}) \mathrm{G}$ conjugated to horseradish peroxidase (HRP) (catalog no., sc-2314; dilution, 1:10,000), donkey anti-goat IgG-HRP (catalog no., sc-2020; dilution, 1:20,000) and goat anti-rabbit IgG-HRP secondary antibodies (catalog no., sc-2004; dilution, 1:5,000)(Santa Cruz Biotechnology, Inc.) conjugated to IRDye ${ }^{\circledR} 800 \mathrm{CW}$ Infrared Dye (LI-COR Biotechnology, Lincoln, NE, USA). Following $1 \mathrm{~h}$ of incubation at $37^{\circ} \mathrm{C}$, the membranes were washed 3 times with TBST. The blots were visualized using the Odyssey ${ }^{\circledR}$ CLx Infrared Imaging system (LI-COR Biotechnology). The signals were densitometrically assessed (Odyssey Application software; version 3.0) and normalized to $\beta$-actin or GAPDH.

Luciferase assays. For the overexpression or suppression of miR-187, 100 pM miR-187 mimics or inhibitors were introduced into $1 \times 10^{6}$ SUDHL2 cells via electroporation $(1,600 \mathrm{~V}, 20 \Omega$, 1 pulse; ECM 830 Square Wave Electroporation System; BTX $^{\circledR}$ Harvard Apparatus, Inc., Holliston, MA, USA). For the luciferase assay, $2 \mu \mathrm{g}$ miR-Report BCL6 3'-UTR plasmid or the vector control were transferred into $1 \times 10^{6}$ SUDHL2 cells using electroporation according to the manufacturer's protocols. Following cell transfection for $24 \mathrm{~h}$, the cells were lysed, and luciferase activities was measured using a Dual-Luciferase ${ }^{\circledR}$ Reporter Assay system (Promega Corporation), according to the manufacturer's protocol. Luciferase activity was measured and normalized to Renilla luciferase activity.

Flow cytometry analysis. Following appropriate treatment, the cultured cells were harvested and washed with phosphate-buffered saline. For each sample, $5 \times 10^{5}$ cells were stained with the Annexin V-FITC/PI Staining Detection kit (Beijing Biosea Biotechnology Co., Ltd.) for $30 \mathrm{~min}$ at room temperature, and were subsequently analyzed using flow cytometry (FACSCanto II; BD Biosciences, Franklin Lakes, NJ, USA).

MTT assay and cell counting kit $8(C C K-8)$ assay. Doxorubicin (DOX), vincristine (VCR) and bortezomib were purchased from Sigma-Aldrich (St. Louis, MO, USA). The SUDHL2 cells were treated with the drugs at concentrations ranging between 
0 and $250 \mathrm{nM}(0,50,100,150,200$ and $250 \mathrm{nM})$ for $48 \mathrm{~h}$; the medium was then discarded, and $20 \mu 10.05 \%$ MTT was added and subsequently incubated for $4 \mathrm{~h}$. The medium was aspirated following centrifugation $(7,200 \mathrm{x} \mathrm{g})$, and dimethyl sulfoxide was added and mixed for $2 \mathrm{~min}$. The $\mathrm{IC}_{50}$ for each drug was detected by a microplate reader (SpectraMax ${ }^{\circledR}$ M5 Microplate Reader; Molecular Devices, LLC, Sunnyvale, CA, USA). For CCK-8 assay, cells were seeded in 96-well plates at a density of 2,000 cells per well. The absorptions of the cells were measured using a CCK-8 kit (Dojindo Molecular Technologies, Inc., Kumamoto, Japan), according to the manufacturer's protocols at different indicated time points.

Statistical analysis. Data are expressed as the mean \pm standard deviation. Comparisons between groups were analyzed using Student's $t$ test or analysis of variance, and the Student-Newman-Keuls method was utilized to estimate the level of significance. $\mathrm{P}<0.05$ was considered to indicate a statistically significant difference.

\section{Results}

Expression pattern and function of miR-187 in B-cell lymphoma cells. To determine whether miR-187 functions in lymphoma development, the expression pattern of miR-187 was analyzed by RT-qPCR in various types of lymphoma cells (SUDHL2, OCI-LY3 and Raji cells). B cells isolated from the healthy donors were used as a control. The results revealed that miR-187 expression was significantly lower in the B-cell lymphoma cells compared with the healthy B cell controls. However, expression patterns appeared to vary depending on the cell type, miR-187 expression levels in the SUDHL2 and OCI-LY3 cells were 7.3 and $16.1 \%$, respectively, of those observed in the healthy $\mathrm{B}$ cells $(\mathrm{P}<0.01)$, whilst the Raji cell miR-187 expression was $57.6 \%$ of that observed in the healthy B cells $(\mathrm{P}<0.05)$ (Fig. 1A).

To study the function of miR-187 in DLBCL cells, its expression was induced using the miR-187 mimics or the scramble control in the SUDHL2 cells via electroporation; RT-qPCR was then utilized to detect miR-187 levels. As shown in Fig. 1B, miR-187 was significantly upregulated by the miR-187 mimics at $24 \mathrm{~h}$ post electroporation $(\mathrm{P}<0.01)$ compared to the controls. To analyze whether miR-187 serves a role in the cell growth of SUDHL2 cells, a CCK-8 assay was performed to assess the proliferation of the parental control SUDHL2 cells and SUDHL2 cells overexpressing miR-187 mimics or scramble control. The results of CCK-8 assay demonstrated that there was no significant difference between the proliferation of the parental control and the miR-187 overexpressing cells (Fig. 1C). However, miR-187 overexpression did affect apoptosis in SUDHL2 cells. SUDHL2 cells that had overexpressed the miR-187 mimic for $48 \mathrm{~h}$ were subjected to flow cytometric analysis to assess the number of apoptotic cells in each treatment group. It was observed that miR-187 overexpression resulted in an increased percentage of apoptotic cells compared with the scramble and parental control groups (Fig. 1D). This result was also verified by western blot analysis, which demonstrated that the cleavage activity of caspase-3 and PARP significantly increased in the SUDHL2 cells overexpressing miR-187 compared with the control groups (Fig. 1E).
Table I. Comparison of $\mathrm{IC}_{50}$ of each group for different drugs.

\begin{tabular}{llll}
\hline & \multicolumn{3}{c}{$\mathrm{IC}_{50}, \mathrm{nmol} / \mathrm{l}($ mean $\pm \mathrm{SD})$} \\
\cline { 2 - 4 } Drug & Control & Scramble & Mimics \\
\hline Doxorubicin & $78 \pm 8.5$ & $82 \pm 10.1$ & $46 \pm 5.4^{\mathrm{a}}$ \\
Bortezomib & $52 \pm 2.1$ & $55 \pm 8.4$ & $46 \pm 4.9^{\mathrm{b}}$ \\
Vincristine & $73 \pm 2.5$ & $69 \pm 10.4$ & $42 \pm 6.1^{\mathrm{a}}$
\end{tabular}

${ }^{\mathrm{a}} \mathrm{P}<0.05$ and ${ }^{\mathrm{b}} \mathrm{P}<0.01$ vs. scramble control. $\mathrm{IC}_{50}$, half maximal inhibitory concentration.

Table II. Comparison of $\mathrm{IC}_{50}$ of each group for different drugs.

\begin{tabular}{|c|c|c|c|}
\hline \multirow[b]{3}{*}{ Drug } & \multicolumn{3}{|c|}{$\mathrm{IC}_{50}, \mathrm{nmol} / \mathrm{l}($ mean $\pm \mathrm{SD})$} \\
\hline & \multirow[b]{2}{*}{ Control } & \multicolumn{2}{|c|}{ miR-187 overexpression } \\
\hline & & Vector & BCL6 OE \\
\hline Doxorubicin & $82 \pm 2.1$ & $79 \pm 6.2$ & $129 \pm 13.9^{a}$ \\
\hline Bortezomib & $42 \pm 2.14$ & $48 \pm 3.5$ & $96 \pm 4.9^{\mathrm{b}}$ \\
\hline Vincristine & $68 \pm 5.8$ & $71 \pm 6.3$ & $157 \pm 12.1^{\mathrm{a}}$ \\
\hline
\end{tabular}

${ }^{\mathrm{a}} \mathrm{P}<0.05$ and ${ }^{\mathrm{b}} \mathrm{P}<0.01$ vs. scramble control. $\mathrm{IC}_{50}$, half maximal inhibitory concentration; miR, microRNA; BCL6 OE, B-cell lymphoma 6 overexpression; Vector, empty vector control.

miR-187 enhances the chemotherapeutic sensitivity of SUDHL2 cells. Drug resistance is a major challenged faced during the treatment of lymphoma $(14,15)$. To evaluate whether miR-187 serves a role in the drug resistance of DLBCL cells, the chemotherapeutic sensitivity of the parental SUDHL2 cells, or SUDHL2 cells overexpressing miR-187 or the scramble control, to 5 drugs used clinically was analyzed via MTT assay. The drugs included DOX, VCR and bortezomib, which were used in testing the chemotherapeutic sensitivity of the cells. The results demonstrated that, following transfection of the miR-187 mimics and scramble control, the capability of multidrug resistance in the SUDHL2 cells. Overexpressing miR-187 significantly decreased the concentrations of DOX between $78 \pm 8.5$ and $46 \pm 5.4(\mathrm{P}<0.05)$, bortezomib between $52 \pm 2.1$ and $46 \pm 4.9(\mathrm{P}<0.01)$ and VCR between $73 \pm 2.5$ and $42 \pm 6.1(\mathrm{P}<0.05)$, suggesting that miR-187 serves an important role in multidrug resistance (Table I).

BCL6 is a target gene of miR-187. To elucidate the detailed molecular mechanisms of miR-187 in DLBCL cells, its potential targets were evaluated using TargetScan (www.targetscan.org), which indicated that there is one miR-187 conserved binding site in the BCL6 3'-UTR (Fig. 2A). In the DLBCL cell lines, western blot analysis revealed that BCL6 was significantly upregulated in the SUDHL2 and OCI-LY3 cells, and slightly upregulated in the Raji cells, whereas there was limited BCL6 expression detected in the $\mathrm{B}$ cell control; this indicated a negative association with miR-187 (Fig. 2B). In addition, western blot analysis indicated that the overexpression of miR-187 
A

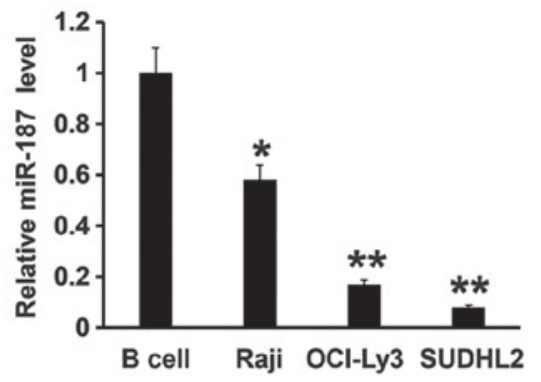

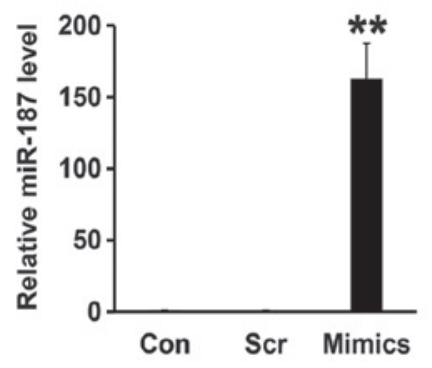

C

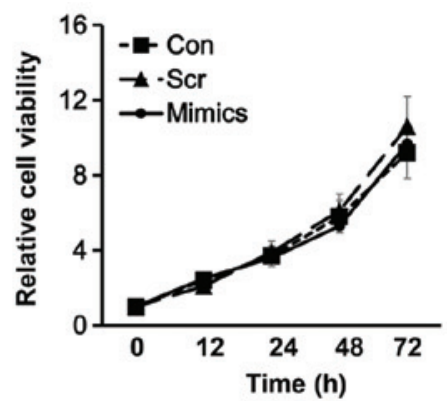

D

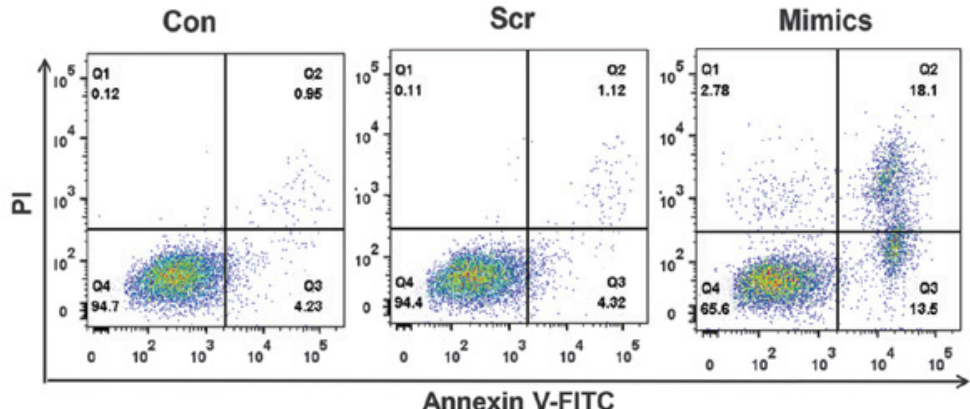

$\mathbf{E}$

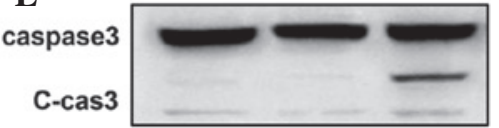

PARP

C-PARP

Actin

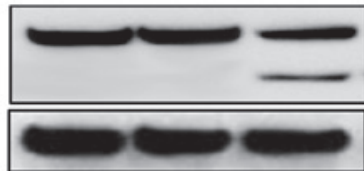

Figure 1. Expression and function of miR-187 in diffuse large B-cell lymphoma cells. (A) Relative expression level of miR-187 was detected via reverse transcription-quantitative polymerase chain reaction in B-cells from healthy donors, Raji cells, OCI-LY3 and SUDHL cells. The U6 gene was used as an endogenous control. (B) Relative expression of miR-187 in Con, SUDHL cells electroporated with $100 \mathrm{pM}$ mimics or Scr negative control for $24 \mathrm{~h}$. All experiments were repeated at least three times. (C) Cell viability was analyzed using CCK-8 assay in the SUDHL cells transfected with scramble or miR-187 mimics compared with the parental control. (D) Flow cytometry assay using annexin V-FITC and PI staining was utilized to detect the apoptosis of the Con cells, SUDHL cells electroporated with $100 \mathrm{pM}$ mimics or Scr negative control for $48 \mathrm{~h}$. (E) Western blot analysis was used to detect the cleavage activity of caspase- 3 and PARP in the SUDHL2 cells with different treatment for $48 \mathrm{~h}$. $\mathrm{P}<0.05$ and ${ }^{* *} \mathrm{P}<0.01$ vs. controls. miR, microRNA; Con, parental control SUDHL cells; Scr, scramble; Mimics, miR-187 mimics; FITC, fluorescein isothiocyanate; PI, propidium iodide; PARP, poly ADP ribose polymerase.

A

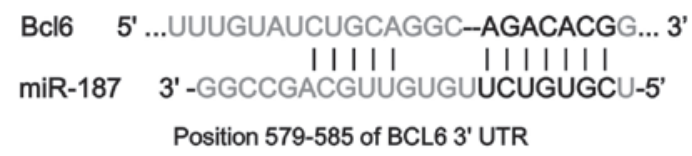

C

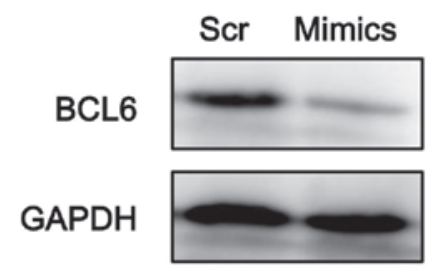

D
B B-Cell Raji OCI-LY3 SUDHL2 BCL6

GAPDH
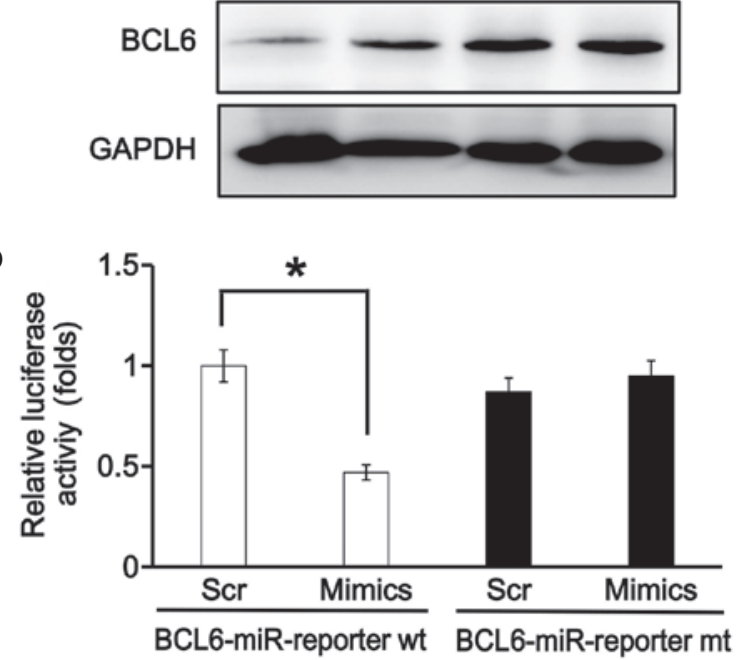

Figure 2. miR-187 directly binds to the 3'-UTR of BCL6 mRNA. (A) miR-187 binding sites on the 3'-UTR of BCL6 mRNA had been predicted by www.microrna.org. Black font indicates the conserved binding sites. (B) Western blotting was utilized to detect the protein level of BCL6 in the B-cells from healthy donors, and the Raji, OCI-LY3 and SUDHL2 cells. GAPDH was used as an endogenous control. (C) BCL6 protein level in the SUDHL2 cells overexpressing miR-187 mimics or scramble control. GAPDH was used as a control. (D) A luciferase reporter assay was performed to detect the binding of miR-187 with the complementary fragment harbouring in the 3'-UTR of BCL6 mRNA. All experiments were repeated at least three times. "P $<0.05$ vs. controls. UTR, untranslated region; BCL6, B-cell lymphoma 6; miR, microRNA; GAPDH, glyceraldehyde 3-phosphate dehydrogenase; Scr, scramble; wt, wild-type; mt, mutant-type.

attenuated BCL6 expression in the SUDHL2 cells (Fig. 2C). The miR-Report luciferase assay was performed to determine whether miR-187 directly targeted the 3'-UTR of BCL6. The luciferase assay indicated that miR-187 overexpression significantly reduced the luciferase activity in the wild-type
BCL6 3'-UTR reporter ( $\mathrm{P}>0.05)$, but not in the mutant reporter (Fig. 2D).

Exogenous BCL6 reverses miR-187 function. In order to determine whether miR-187 regulates apoptosis and 
A

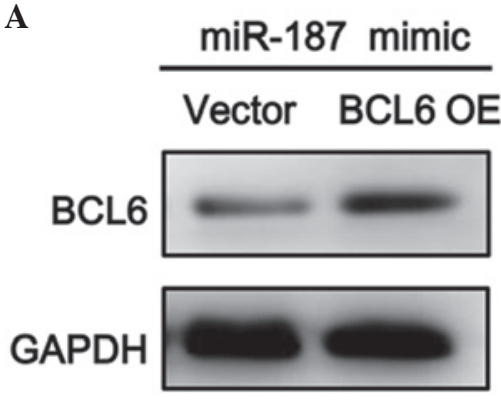

B

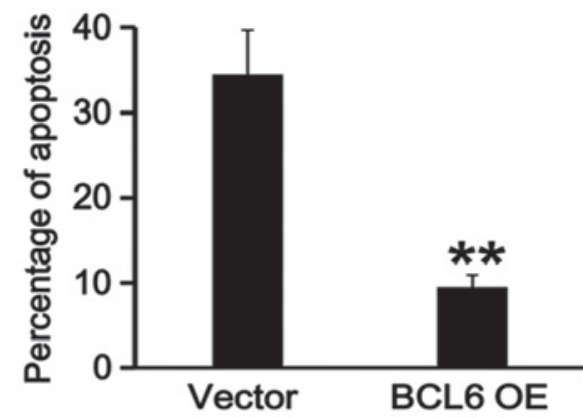

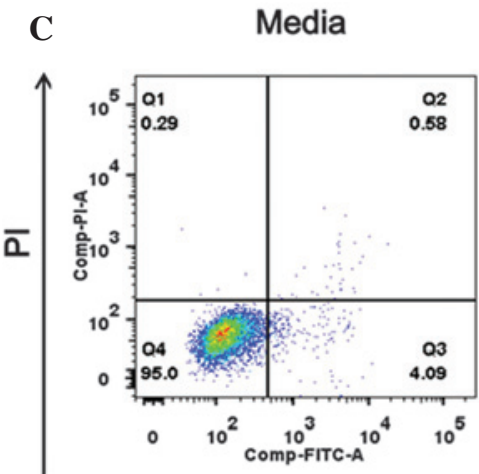

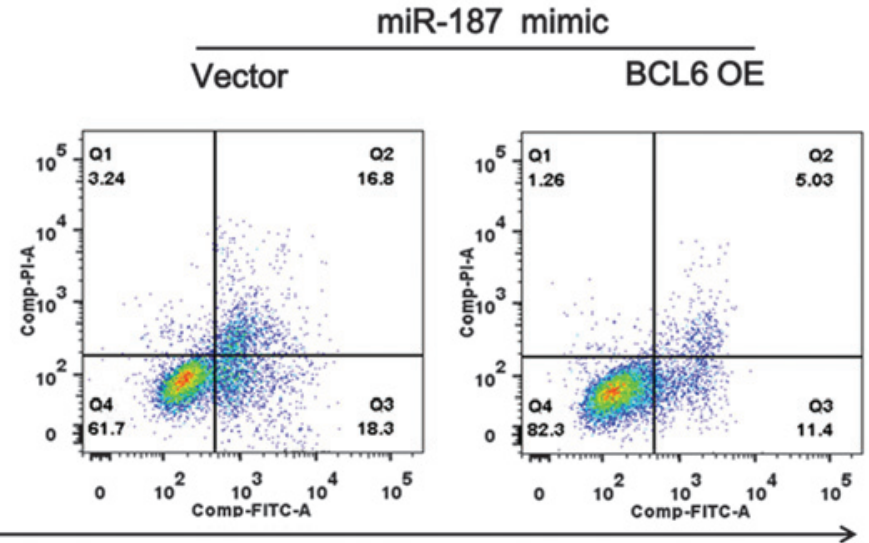

Annexin V-FITC

Figure 3. Exogenous expression of BCL6 in the SUDHL2 cells reversed the miR-187 mimic function. (A) Western blotting was utilized to detect the protein level of BCL6 in the miR-187 overexpressing SUDHL cells transfected with BCL6 OE or a vector control (vector). (B) Percentage of apoptotic cells was counted in miR-187 overexpressing SUDHL cells transfected with BCL6 OE or a vector control. All experiments were repeated at least three times. ${ }^{* *} \mathrm{P}<0.01 \mathrm{vs}$. controls. (C) A flow cytometry assay, using annexin V-FITC and PI staining, was performed to detect the apoptotic cells co-transfected in the miR-187 overexpressing SUDHL cells transfected with BCL6 OE or vector control (vector). BCL6 OE, BCL6 overexpressing plasmid; BCL6, B-cell lymphoma 6; miR, microRNA; Annexin V-FITC, Annexin V-fluorescein isothiocyanate; PI, propidium iodide; GAPDH, glyceraldehyde 3-phosphate dehydrogenase; Comp-PI-A, propidium iodide; Comp-FITC-A, Annexin V-fluorescein isothiocyanate.

multidrug resistance through its target BCL6, miR-187 mimics and BCL6 overexpression plasmids were co-transfected into the SUDHL2 cells. Western blot analysis indicated that the BCL6 downregulation induced by miR-187 was completely reversed by exogenous BCL6, as the BCL6 overexpression plasmid lacked miR-187 binding sites (Fig. 3A). Subsequently, flow cytometry was performed, and the results demonstrated that, although there was abundant miR-187 in the DLBCL cells, BCL6 overexpression continued to inhibit cell apoptosis (Fig. 3B and $\mathrm{C})$. Furthermore, the suppression of multidrug resistance capability induced by miR-187 was significantly reversed following BCL6 overexpression (Table II). In the absence of the miR-187 and BCL6 3'-UTR interaction, the effect of miR-187 was diminished, thus suggesting that miR-187 mediates apoptosis and multidrug resistance through its transcriptional modulation of BCL6.

\section{Discussion}

Over the past decade, miRNAs have gained increasing attention from researchers regarding their possible role in cancer biology, with a number of miRNAs being demonstrated to serve crucial regulatory roles in cancer development and tumorigenesis. Based on the accumulating data, it has been debated as to whether miRNAs may serve as novel therapeutic targets for numerous malignancies (16).

Research regarding the biological function of $\mathrm{miR}-187$ in carcinogenesis remains in its infancy. Studies have implicated miR-187 activity in the development of various types of tumors $(5,17,18)$; however, its role in DLBCL has not yet been established.

The BCL6 protein, a zinc finger transcription factor that has been conserved throughout evolution, has demonstrated high expression levels in numerous types of human cancer, alongside lymphoid system malignancies (19). Further studies have observed potent BCL6 protein expression in various types of human cancer outside of the lymphoid system. For example, overexpression of BCL6 protein was demonstrated in breast cancer tissues, particularly in high-grade ductal breast cancer, when compared to normal mammary gland tissues $(20,21)$. Overall, the specific function of BCL6 protein in carcinogenesis remains to be clarified.

The majority of germinal center B-cell-like (GCB) and activated B-cell-like type DLBCL cells are dependent upon BCL6 in order to maintain their proliferation and survival, reflecting the function of BCL6 in GCB DLBCL and supporting the notion that it is a broadly relevant therapeutic target in DLBCL (22-24).

The effect of miR-187 on apoptosis and multidrug resistance in DLBCL cells is most likely achieved through BCL6 
regulation. The following evidence supports this hypothesis: i) The expression of miR-187 is negatively correlated with BCL6 expression in DLBCL cell lines; and ii) BCL6 is a target of miR-187, with miR-187 indirectly promoting lymphoma cell apoptosis and enhancing multidrug sensitivity by targeting its 3'-UTR.

To the best of our knowledge, there are no studies currently published that report of a direct involvement of miR-187 and BCL6 in DLBCL carcinogenesis, thus warranting further investigation of their possible underlying mechanisms in DLBCL. The results of the present study provide evidence that miRNAs may be useful for future development of novel therapeutic strategies for DLBCL. Further studies, possibly utilizing animal models, alongside additional human samples, are required to validate the results from the present study, aiming to further elucidate the functions of miRNA and aid the treatment of lymphoma.

\section{References}

1. Sabattini E, Bacci F, Sagramoso C and Pileri SA: WHO classification of tumours of haematopoietic and lymphoid tissues in 2008: An overview. Pathologica 102: 83-87, 2010.

2. Lenz G and Staudt LM: Aggressive lymphomas. NEngl J Med 362: $1417-1429,2010$

3. Zhou C, Lu Y and Li X: miR-339-3p inhibits proliferation and metastasis of colorectal cancer. Oncol Lett 10: 2842-2848, 2015.

4. Vicente R, Noël D, Pers YM, Apparailly F and Jorgensen C: Deregulation and therapeutic potential of microRNAs in arthritic diseases. Nat Rev Rheumatol: 2015 (Epub ahead of print).

5. Zhao J, Lei T, Xu C, Li H, Ma W, Yang Y, Fan S and Liu Y: MicroRNA-187, down-regulated in clear cell renal cell carcinoma and associated with lower survival, inhibits cell growth and migration though targeting B7-H3. Biochem Biophys Res Commun 438: 439-444, 2013.

6. Casanova-Salas I, Rubio-Briones J, Calatrava A, Mancarella C, Masiá E, Casanova J, Fernández-Serra A, Rubio L, Ramírez-Backhaus M, Armiñán A, et al: Identification of miR-187 and miR-182 as biomarkers of early diagnosis and prognosis in patients with prostate cancer treated with radical prostatectomy. J Urol 192: 252-259, 2014.

7. Locke JM, da Silva Xavier G, Dawe HR, Rutter GA and Harries LW: Increased expression of miR-187 in human islets from individuals with type 2 diabetes is associated with reduced glucose-stimulated insulin secretion. Diabetologia 57: 122-128, 2014.

8. Chang CC, Ye BH, Chaganti RS and Dalla-Favera R: BCL-6, a $\mathrm{POZ/zinc-finger} \mathrm{protein,} \mathrm{is} \mathrm{a} \mathrm{sequence-specific} \mathrm{transcriptional}$ repressor. Proc Natl Acad Sci USA 93: 6947-6952, 1996.

9. Jardin F, Ruminy P, Bastard C and Tilly H: The BCL6 proto-oncogene: A leading role during germinal center development and lymphomagenesis. Pathol Biol (Paris) 55: 73-83, 2007.

10. Duan S, Cermak L, Pagan JK, Rossi M, Martinengo C, di Celle PF, Chapuy B, Shipp M, Chiarle R and Pagano M: FBXO11 targets BCL6 for degradation and is inactivated in diffuse large B-cell lymphomas. Nature 481: 90-93, 2012.
11. Pasqualucci L, Bereshchenko O, Niu H, Klein U, Basso K, Guglielmino R, Cattoretti G and Dalla-Favera R: Molecular pathogenesis of non-Hodgkin's lymphoma: The role of Bcl-6. Leuk Lymphoma 44 (Suppl 3): S5-S12, 2003.

12. Ye BH: BCL-6 in the pathogenesis of non-Hodgkin's lymphoma. Cancer Invest 18: 356-365, 2000

13. Anstaett OL, Brownlie J, Collins ME and Thomas CJ: Validation of endogenous reference genes for RT-qPCR normalisation in bovine lymphoid cells (BL-3) infected with Bovine Viral Diarrhoea Virus (BVDV). Vet Immunol Immunopathol 137: 201-207, 2010.

14. Jiang N, Chen W, Zhang JW, Li Y, Zeng XC, Zhang T, Fu BS, Yi HM and Zhang Q: Aberrantly regulated dysadherin and B-cell lymphoma 2/B-cell lymphoma 2 -associated X enhances tumorigenesis and DNA targeting drug resistance of liver cancer stem cells. Mol Med Rep 12: 7239-7246, 2015.

15. Camicia R, Winkler HC and Hassa PO: Novel drug targets for personalized precision medicine in relapsed/refractory diffuse large B-cell lymphoma: A comprehensive review. Mol Cancer 14: 207,2015

16. Soriano A, Jubierre L, Almazán-Moga A, Molist C, Roma J, de Toledo JS, Gallego S and Segura MF: MicroRNAs as pharmacological targets in cancer. Pharmacol Res 75: 3-14, 2013.

17. Mulrane L, Madden SF, Brennan DJ, Gremel G, McGee SF, McNally S, Martin F, Crown JP, Jirström K, Higgins DG, et al: miR-187 is an independent prognostic factor in breast cancer and confers increased invasive potential in vitro. Clin Cancer Res 18: 6702-6713, 2012.

18. Chao A, Lin CY, Lee YS, Tsai CL, Wei PC, Hsueh S, Wu TI, Tsai CN, Wang CJ, Chao AS, et al: Regulation of ovarian cancer progression by microRNA-187 through targeting Disabled homolog-2. Oncogene 31: 764-775, 2012.

19. Wu Q, Liu X, Yan H, He YH, Ye S, Cheng XW, Zhu GL, Wu WY, Wang XN, Kong XJ, et al: B-cell lymphoma 6 protein stimulates oncogenicity of human breast cancer cells. BMC Cancer 14: 418,2014

20. Bajalica-Lagercrantz S, Piehl F, Farnebo F, Larsson C and Lagercrantz J: Expression of the BCL6 gene in the pre- and postnatal mouse. Biochem Biophys Res Commun 247: 357-360, 1998

21. Bos R, van Diest PJ, van der Groep P, Greijer AE, Hermsen MA, Heijnen I, Meijer GA, Baak JP, Pinedo HM, van der Wall E and Shvarts A: Protein expression of B-cell lymphoma gene 6 (BCL-6) in invasive breast cancer is associated with cyclin D1 and hypoxia-inducible factor-1alpha (HIF-1alpha). Oncogene 22: 8948-8951, 2003.

22. Polo JM, Dell'Oso T, Ranuncolo SM, Cerchietti L, Beck D, Da Silva GF, Prive GG, Licht JD and Melnick A: Specific peptide interference reveals BCL6 transcriptional and oncogenic mechanisms in B-cell lymphoma cells. Nat Med 10: 1329-1335, 2004.

23. Polo JM, Juszczynski P, Monti S, Cerchietti L, Ye K, Greally JM, Shipp M and Melnick A: Transcriptional signature with differential expression of BCL6 target genes accurately identifies BCL6-dependent diffuse large B cell lymphomas. Proc Natl Acad Sci USA 104: 3207-3212, 2007.

24. Cerchietti LC, Yang SN, Shaknovich R, Hatzi K, Polo JM, Chadburn A, Dowdy SF and Melnick A: A peptomimetic inhibitor of BCL6 with potent antilymphoma effects in vitro and in vivo. Blood 113: 3397-3405, 2009. 